\title{
Damage identification of a reinforced concrete frame by finite element model updating using damage parameterization
}

\author{
Sheng-En Fang , Ricardo Perera, Guido De Roeck \\ Department of Structural Mechanics and Industrial Constructions, Universidad Politécnica de Madrid, Madrid 28006, Spain \\ Department of Civil Engineering, Central South University, Changsha, Hunan Province 410075, PR China \\ Division of Structural Mechanics, Department of Civil Engineering, Katholieke Universiteit Leuven, \\ Kasteelpark Arenberg 40, B-3001 Heverlee, Belgium
}

\begin{abstract}
This paper develops a sensitivity-based updating method to identify the damage in a tested reinforced concrete (RC) frame modeled with a two-dimensional planar finite element (FE) by minimizing the discrepancies of modal frequencies and mode shapes. In order to reduce the number of unknown variables, a bidimensional damage (element) function is proposed, resulting in a considerable improvement of the optimization performance. For damage identification, a reference FE model of the undamaged frame divided into a few damage functions is firstly obtained and then a rough identification is carried out to detect possible damage locations, which are subsequently refined with new damage functions to accurately identify the damage. From a design point of view, it would be useful to evaluate, in a simplified way, the remaining bending stiffness of cracked beam sections or segments. Hence, an RC damage model based on a static mechanism is proposed to estimate the remnant stiffness of a cracked $\mathrm{RC}$ beam segment. The damage model is based on the assumption that the damage effect spreads over a region and the stiffness in the segment changes linearly. Furthermore, the stiffness reduction evaluated using this damage model is compared with the FE updating result. It is shown that the proposed bidimensional damage function is useful in producing a well-conditioned optimization problem and the aforementioned damage model can be used for an approximate stiffness estimation of a cracked beam segment.
\end{abstract}

\section{Introduction}

Damage assessment and condition evaluation of existing civil infrastructures are essential for decision making in regular structural maintenance and disaster remediation. Considerable attention has been paid to nondestructive damage identification and structural health monitoring of civil engineering structures during past decades. Damage produces stiffness reduction in damaged structures resulting in changes of structural static and dynamic responses, which means damage can be identified through dynamic techniques, as well as static ones. Various experimental approaches such as modal testing and controlled load-deflection testing as well as indices resulting from both tests should be integrated for a reliable structural condition 
assessment. This paper proposes and investigates dynamic and static approaches validated on an RC structure tested under an alternating combination of static loading tests for damage producing and modal tests.

Owing to the limitation of visual or local experimental techniques global damage identification methods based on the vibration characteristics, which are usually represented as modal parameters, of the structures have been widely investigated and successfully applied in many practical cases updating methods, which overcome the limitations of the direct methods

. Recently, sensitivity-based FE damage identification purposes. These methods aim to update the physical parameters of structures (Young's modulus, density, geometric dimensions, etc.), which generally present physically meaningful results . And the updating process can be achieved by iterative optimization algorithms between objective residuals and model properties. Friswell and Mottershead due to the nonlinear relation elaborate on relevant FE model updating techniques and a comprehensive review of sensitivity-based updating methods is given by Link

In this study, a sensitivity-based FE updating method is developed to identify the damage of a laboratoryscale reinforced concrete (RC) frame. The updating is implemented by the nonlinear least square (LS) method improved by the trust region Newton algorithm. In order to reduce the number of updating parameters, a bidimensional damage function method is proposed for structures modeled with bidimensional finite elements (FEs), which simultaneously improves the iteration performance of the optimization process. Each damage function represents one damage element consisting of some neighboring FEs. Furthermore, the damage function can be simplified into a step alike damage function when necessary, which is applied in this RC frame case. On damage identification of the RC frame, the initially developed FE model divided into several substructures is firstly updated to the reference state using the measured modal frequencies and mode shapes of the undamaged frame. Then a subsequent updating process is performed for a rough estimate of possible damage locations using the modal data of the damaged frame. Finally, the possible damaged regions are fractionized into new substructures (new damage functions) and the reference model is updated again to the damage state. Thus, a more accurate identification of the damage is obtained by comparing the reference and damage states. Then a damage identification process by dynamic means is achieved.

On the other hand, from a design point of view, it would be useful to evaluate, in a simplified way, the remaining bending stiffness of cracked beam sections or segments for further condition assessment or remnant life evaluation of RC structures. But in the existing European and American codes for the design of concrete structures, there are no formulas to estimate the remnant bending stiffness of cracked beam sections or segments. Hence, with an assumption of damage effect spreading over a region of structure, an RC damage model is proposed to estimate the remnant stiffness and to give a general evaluation of the damage situation of a cracked beam segment of the RC frame. An equivalent stiffness formula is deduced using the damage model. Then the remnant stiffness of the cracked beam segment is obtained using a proposed formula based on the damage model and verified against the FE updating results. The relative stiffness (Young's modulus) reductions are compared due to the fact that the proposed formula is suitable for a static mechanism, but the updating results are related to a dynamic mechanism. It is shown that the remnant stiffness of the cracked beam segment can be well estimated using the proposed damage model and this model is applicable to similar cases.

\section{FE model updating method}

In general, sensitivity-based FE model updating can be regarded as an optimization process aimed at minimizing the discrepancies between the measured and analytical model properties. This section describes the theory of the updating method used in this study.

\subsection{Objective function}

Two modal parameters, modal frequency and mode shape are adopted to construct the residual vectors of the objective function, which is stated as a nonlinear LS problem:

$$
f(\mathbf{u})=\frac{1}{2}\|\mathbf{r}(\mathbf{u})\|^{2}=\frac{1}{2}\left\|\begin{array}{c}
\mathbf{r}_{f}(\mathbf{u}) \\
\mathbf{r}_{m}(\mathbf{u})
\end{array}\right\|^{2} \quad\left\{\begin{array}{c}
\mathbf{r}: \mathbb{R}^{n} \Rightarrow \mathbb{R}^{m} \\
\mathbf{u} \in \mathbb{R}^{n}
\end{array}\right\} \quad \text { with } \min _{u} f(\mathbf{u}),
$$


where $\|\cdot\|$ denotes the Euclidean norm, u denotes the vector containing the unknown updating parameters, $\mathbf{r}$ represents the residual vector comprising the frequency residual $\mathbf{r}_{f}$ and the mode shape residual $\mathbf{r}_{m}$ :

$$
\mathbf{r}_{f}(\mathbf{u})=\frac{\lambda_{i}^{A}(\mathbf{u})-\lambda_{i}^{E}}{\lambda_{i}^{E}}, \quad \mathbf{r}_{m}(\mathbf{u})=\phi_{i}^{A}(\mathbf{u})-\phi_{i}^{E}
$$

in which $\lambda_{i}^{A}$ and $\lambda_{i}^{E}$ are the square of the analytical and measured circular frequencies $\left(\lambda=(2 \pi f)^{2}\right)$, respectively; $\phi_{i}^{A}$ and $\phi_{i}^{E}$ are the mass-normalized analytical and measured mode shapes, respectively. It should be mentioned that in this study, the measured mode shapes are also mass normalized because the impact force was simultaneously recorded in the RC frame experiment. Alternatively, the mass matrix of the analytical model might be used for mass normalization of the measured mode shapes [15].

Meanwhile, the residuals can be weighted according to their importance and the accuracy level of measurement when necessary. A weighted minimization problem can be defined as

$$
\min \frac{1}{2}\left\|\mathbf{W}^{1 / 2} \mathbf{r}(\mathbf{u})\right\|^{2}
$$

with the weighting matrix $\mathbf{W}$.

\subsection{Updating parameter and correction factor}

Young's modulus of concrete, $E$, which alternatively reflects the section stiffness as the section inertia $I$ does, is selected to be the updating parameter in this analysis. A correction factor is used to represent the change of Young's modulus. A dimensionless correction factor $C_{E, i}$ is used herein instead of an absolute value in order to avoid the different orders of different parameters in their magnitudes:

$$
C_{E, i}=-\frac{E_{i}^{\mathrm{upd}}-E_{i}^{\mathrm{ref}}}{E_{i}^{\mathrm{ref}}},
$$

where $E_{i}^{\text {upd }}$ and $E_{i}^{\text {ref }}$ are the updated and reference values of Young's modulus of substructure $i$, respectively. Accordingly, the updated modulus is $E_{i}^{\mathrm{upd}}=E_{i}^{\mathrm{ref}}\left(1-C_{E, i}\right)$, where a positive value of the correction factor means a reduction of Young's modulus.

\subsection{Bidimensional damage function}

Adjusting the stiffness property of each element separately is prone to generate numerous independent unknown variables, which frequently results in an ill-conditioned optimization problem and considerably increases the computational expense. In order to reduce the number of unknown variables, Teughels et al. [16] proposed an additional parameterization method, named damage function, assuming that the correction factors of updating parameters vary continuously over the FE model. This method was successfully implemented on an RC concrete beam modeled with one-dimensional beam elements to identify the damage. In this study, a bidimensional damage function is proposed as an extension of the aforementioned onedimensional damage function method for the cases with structures modeled with two-dimensional (2D) FEs.

As an illustration, the proposed bidimensional damage function is constructed using the linear nodal shape functions defined on the standard 2D rectangular FE with four nodes, which is used to establish the FE model of the RC frame. The element-level local damage function, $N_{i}^{\text {de }}(x, y)(i=1,2,3,4)$, is defined as (Fig. 1(a))

$$
\begin{array}{ll}
N_{1}^{\mathrm{de}}(x, y)=\frac{1}{4}(1-x)(1-y), & N_{2}^{\mathrm{de}}(x, y)=\frac{1}{4}(1+x)(1-y), \\
N_{3}^{\mathrm{de}}(x, y)=\frac{1}{4}(1+x)(1+y), & N_{4}^{\mathrm{de}}(x, y)=\frac{1}{4}(1-x)(1+y)
\end{array}
$$

with $x, y$ define the geometrical coordinates of FE $e$.

Then the global damage function $\mathbf{N}_{i}(x, y)$ linked to a damage element or its nodes can be constructed by mapping $N_{1}^{\mathrm{de}}(x, y)$ onto damage elements consisting of a set of neighboring FEs:

$$
\mathbf{N}_{i}(x, y)=\bigcup_{\mathrm{de}=1}^{n_{\mathrm{de}}} b_{\mathrm{de}} N_{i}^{\mathrm{de}}(x, y) \quad\left(b_{\mathrm{de}}=\left\{\begin{array}{cc}
1 & \text { if } i \in \mathrm{de} \\
0 & \text { else }
\end{array}\right) \quad(U \Rightarrow \text { assembly symbol }),\right.
$$


(a)

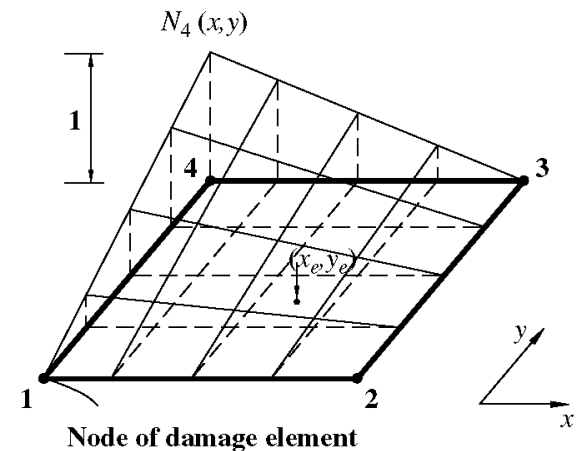

(b)

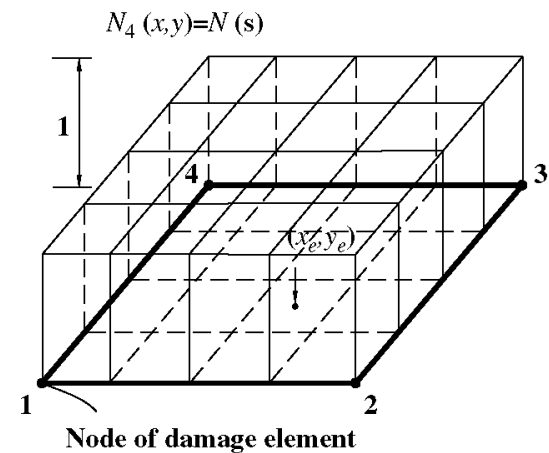

Fig. 1. Bidimensional (a) linear (b) step damage function.

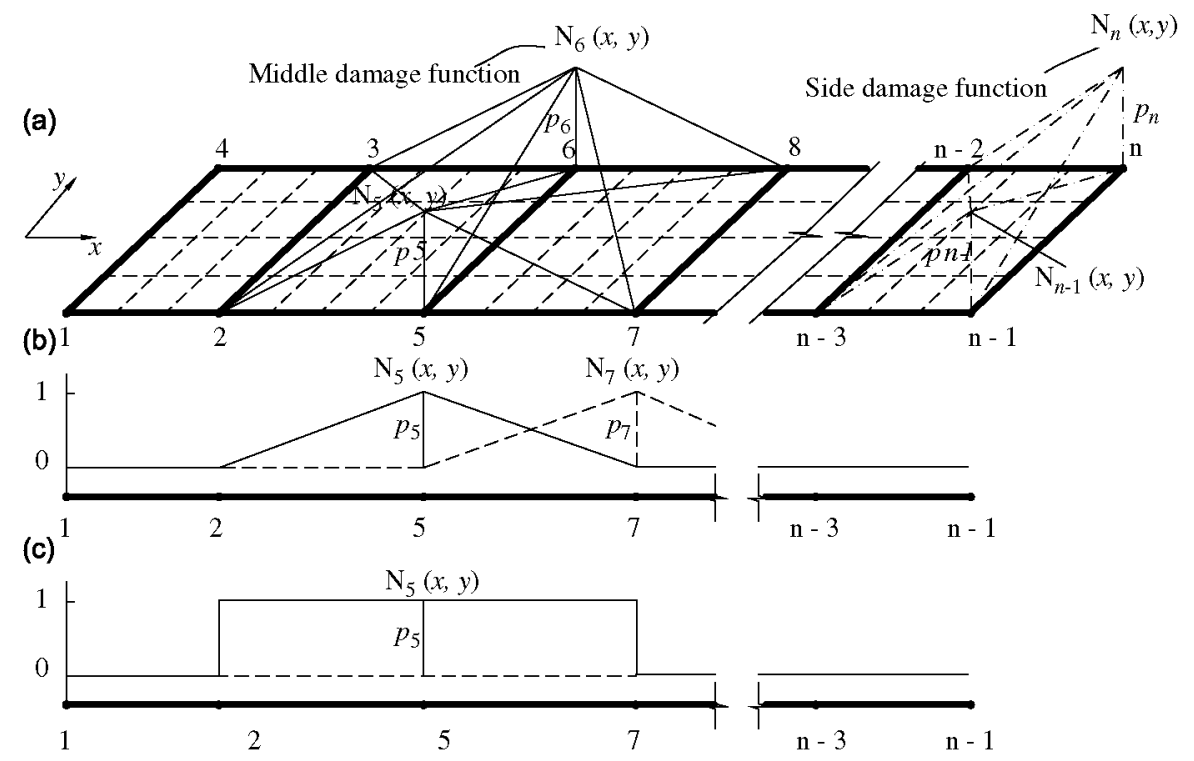

Fig. 2. Global damage function defined on a planar deck: (a, b) middle and edge linear functions and (c) step function.

where $n_{\mathrm{de}}$ is the number of damage elements, $i$ denotes a node of certain FE and de denotes a specific damage element.

As an example, Fig. 2(a) shows the damage-element mesh of a deck modeled with planar rectangular FEs. The global damage function of this mesh is defined with respect to the nodes (or elements). Each $\mathbf{N}_{i}(x, y)$ differs from zero only in its adjacent damage elements and equals zero in all the other damage elements. $\mathbf{N}_{i}(x, y)$ is scaled to be $\left|\mathbf{N}_{i}(x, y)\right|=1$ at the side nodes of damage elements, which results in a pyramid global damage function as shown in Fig. 2.

With the application of the pre-described damage function, the damage situation of a FE $e$ can be evaluated by a correction factor $c^{e}$ defined as a linear combination of all the global damage functions:

$$
c^{e}=\sum_{i=1}^{n_{\mathrm{de}}} p_{i} \mathbf{N}_{i}\left(x_{e}, y_{e}\right),
$$

where $c^{e}$ denotes the correction factor of $e$ with the global coordinates $x_{e}, y_{e}$ at the center point of $e$ inside the damage element $j$ (the continuous function $c^{e}$ is discretized at the FE's center, which means a constant 
correction value is assigned for each updating $\mathrm{FE}), p_{i}$ is the multiplication factor of $\mathbf{N}_{i}(x, y)$ and its vector $\mathbf{p}$ replaces the vector $\mathbf{u}$ in Eq. (1) once the damage function method is used.

When written in a full-length matrix, the correction factor matrix $\mathbf{C}$ can be defined as

$$
\mathbf{C}_{n_{e} \times 1}=[\mathbf{N}(x, y)]_{n_{e} \times n_{p}} \mathbf{p}_{n_{p} \times 1} \Leftrightarrow\left\{\begin{array}{c}
c^{1} \\
c^{2} \\
\vdots \\
c^{n_{e}}
\end{array}\right\}=\left[\begin{array}{cccc}
\mathbf{N}_{1}\left(x_{1}, y_{1}\right) & \mathbf{N}_{2}\left(x_{1}, y_{1}\right) & \ldots & \mathbf{N}_{n_{p}}\left(x_{1}, y_{1}\right) \\
\mathbf{N}_{1}\left(x_{2}, y_{2}\right) & \mathbf{N}_{2}\left(x_{2}, y_{2}\right) & \ldots & \mathbf{N}_{n_{p}}\left(x_{2}, y_{2}\right) \\
\vdots & \vdots & \ldots & \vdots \\
\mathbf{N}_{1}\left(x_{n_{e}}, y_{n_{e}}\right) & \mathbf{N}_{2}\left(x_{n_{e}}, y_{n_{e}}\right) & \ldots & \mathbf{N}_{n_{p}}\left(x_{n_{e}}, y_{n_{e}}\right)
\end{array}\right]\left\{\begin{array}{c}
p_{1} \\
p_{2} \\
\vdots \\
p_{n_{p}}
\end{array}\right\} .
$$

It can be seen from Eq. (7) and Fig. 2 that the application of damage function guarantees a smooth distribution of updating parameters and $p_{i}$ turns out to be the actual updating variable of an optimization problem. Accordingly, the construction of the multidimensional damage function for planar triangular FE and three-dimensional cases can be achieved using similar procedures as prescribed.

In this study, only one model parameter, Young's modulus of concrete, is updated and the parameter adjustment is based on a substructure-level updating, which means the frame model is divided into several substructures and all the FEs in each substructure have the same correction factor. Hence, the proposed damage function is reconstructed and simplified into a bidimensional step function $N_{i}(s)$ equal to 1 over the damage element, as shown in Figs. 1 and 2(b):

$$
\begin{gathered}
N_{i}^{\mathrm{de}}(x, y)=N_{i}(s)=1 \quad \text { (local function) } \\
\mathbf{N}_{i}(x, y)=\bigcup_{\mathrm{de}=1}^{n_{\mathrm{de}}} b_{\mathrm{de}} N_{i}^{\mathrm{de}}(x, y)=\mathbf{N}_{i}(s)=\bigcup_{\mathrm{de}=1}^{n_{\mathrm{de}}} b_{\mathrm{de}} N_{i}(s) \quad \text { (global function). }
\end{gathered}
$$

Then the corresponding correction factor $C_{j}$ of the substructure $j$ is

$$
C_{j}=\sum_{i=1}^{n_{5}} p_{i} \mathbf{N}_{i}(s)
$$

Here, $c^{e}$ is replaced by $C_{j}$ representing a substructure-level correction factor and $n_{\mathrm{de}}$ is replaced by $n_{s}$ which is the number of substructures. Eq. (9) presents a stepwise function instead of a piecewise linear function as shown in Eq. (6).

Using damage function considerably reduces the number of unknown variables in the updating process and hence guarantees smooth and physically meaningful results. Meanwhile, the accuracy of updating results can be improved by using a finer damage-element mesh or using a parameterized damage function instead of the fixed piecewise linear function.

\subsection{Optimization algorithm and sensitivity matrix}

The residual vector $\mathbf{r}$ generally has a nonlinear relation with unknown model properties and thus the objective function $f(\mathbf{u})$ is minimized using a standard Trust Region Newton algorithm . In each iteration step s, the searching steps are limited within a 'trust region' to avoid unexpected large steps and $f(\mathbf{u})$ is approximated by a quadratic minimizor $m(z)$ defined by the truncated Taylor series of $f(\mathbf{u})$, which is $f_{s}$ in the following equation:

$$
\min m(z)=f_{s}+\left[\nabla f_{s}\right]^{\mathrm{T}} z+\frac{1}{2} z^{\mathrm{T}}\left[\nabla^{2} f_{s}\right] z \quad\left(\|z\| \leqslant A_{u}\right),
$$

where $z$ is the step vector and the gradient and Hessian of $f(\mathbf{u}), \nabla f_{s}$ and $\nabla^{2} f_{s}$, are defined as

$$
\nabla f_{s}=\nabla f(\mathbf{u})=\sum_{i=1}^{m} r_{i}(\mathbf{u}) \nabla r_{i}(\mathbf{u})=J_{u}(\mathbf{u})^{\mathrm{T}} r(\mathbf{u}),
$$




$$
\nabla^{2} f_{s}=\nabla^{2} f(\mathbf{u})=J_{u}(\mathbf{u})^{\mathrm{T}} J_{u}(\mathbf{u})+\sum_{i=1}^{m} r_{i}(\mathbf{u}) \nabla^{2} r_{i}(\mathbf{u}) \approx J_{u}(\mathbf{u})^{\mathrm{T}} J_{u}(\mathbf{u}),
$$

where $J_{u}(\mathbf{u})$ is the sensitivity matrix consisting of the first partial derivatives of $r_{i}(\mathbf{u})$.

With the derivatives of $\mathbf{r}$ with respect to the correction factor $c$ [18],

$$
\frac{\partial r_{f}}{\partial c}=\frac{1}{\lambda_{i}^{E}} \frac{\lambda_{i}^{A}}{\partial c} \text { and } \quad \frac{\partial r_{m}}{\partial c}=\frac{\phi_{i}^{A}}{\partial c}
$$

the sensitivity of $\mathbf{r}$ with respect to the updating parameter $p_{i}$ can be deduced in a component notation as

$$
\frac{\partial r_{j}}{\partial p_{i}}=\sum_{e=1}^{n_{e}} \frac{\partial r_{j}}{\partial c^{e}} \frac{\partial c^{e}}{\partial p_{i}}=\sum_{e=1}^{n_{e}} \frac{\partial r_{j}}{\partial c^{e}} N_{i, e}\left(x_{e}, y_{e}\right) .
$$

And after introducing the damage function, the sensitivity (Jacobian) matrix is reduced to $\mathbf{J}_{r}$ :

$$
\left[\mathbf{J}_{r}\right]_{m \times q}=\left[\mathbf{J}_{o}\right]_{m \times n_{e}}[\mathbf{N}]_{n_{e} \times q} \quad\left(m, q \ll n_{e}\right),
$$

where $m$ denotes the number of residual vectors and $q$ denotes the number of actual variables of the optimization problem.

It can be seen from Eq. (14) that the dimension of sensitivity matrix is considerably reduced and thus increasing the possibility of obtaining a well-conditioned optimization problem.

\section{Damage model of an RC beam segment}

When cracked, RC undergoes complicated static and dynamic mechanisms. The stress and strain distributions of concrete and reinforcement are complicated especially in the cracked regions, which makes an accurate stiffness estimation of a cracked section difficult, to say nothing of a cracked segment. However, in order to assess the conditions of RC structures, it would be very useful to estimate the remnant stiffness of cracked structural components by some indices. For example, a damage coefficient, $d$, evaluated as the ratio between the stiffness reductions in the damaged zone and the initial undamaged stiffness:

$$
d=1-\frac{E I^{d}}{E I^{u}}
$$

where $E I^{u}$ and $E I^{d}$ are the bending stiffness values of intact and damaged sections or components, respectively. This damage coefficient is comparative to the correction factor defined in Eq. (6) when they are applied between undamaged and damaged structures.

However, in spite of its importance, in the existing codes for the design of concrete structures, no formula is provided to estimate the remnant stiffness of cracked components of RC structures under bending moments. Hence, as a supplement of the FE updating techniques for damage identification, a simple and practical RC damage model is proposed to approximately estimate the remnant stiffness of a cracked beam segment under bending moment in this section. And the obtained value can subsequently be compared with that estimated from the dynamic identification procedure. To achieve this, an expression for estimating the stiffness of a cracked beam section is firstly deduced and then the formula for the remnant stiffness evaluation of a cracked beam segment is established.

\subsection{Stiffness estimation for a cracked beam section}

To estimate the stiffness of a cracked beam section, a theoretical analysis of a cracked rectangular RC beam section is presented. Some basic assumptions of neglecting the tensile strength of concrete, neglecting the contribution of the compression reinforcement due to its little influence under moderate loads and the cracked sections remaining plane were adopted herein. In Fig. 3(a), by equilibrium of internal moments across the 


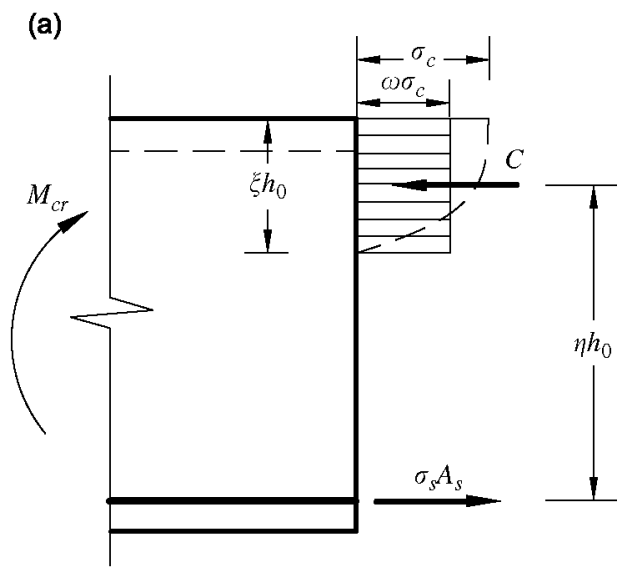

(b)
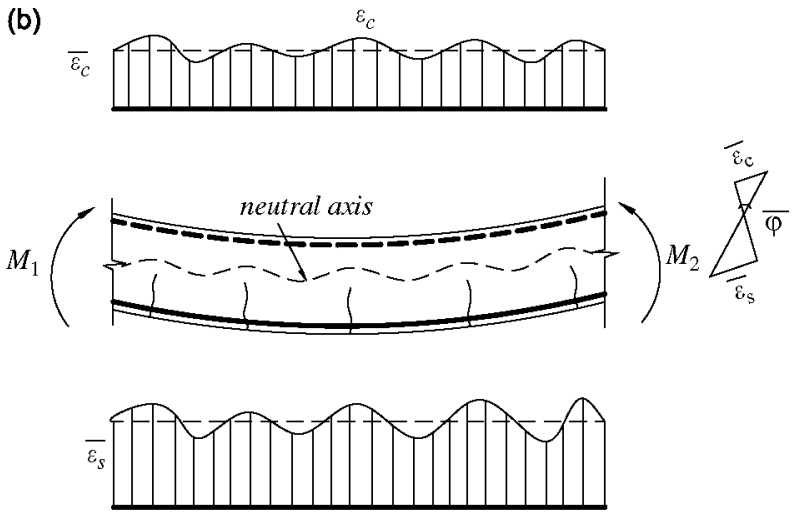

Fig. 3. Computational model of a cracked RC beam cross-section and segment: (a) stress distribution of cracked cross-section and (b) strain distribution of concrete and reinforcement in the segment.

cracked cross-section the following expressions are obtained:

$$
M_{\mathrm{cr}}=C \eta h_{0}=\omega \sigma_{c} \xi h_{0} b \eta h_{0} \Rightarrow \sigma_{c}=\frac{M_{\mathrm{cr}}}{\omega \xi \eta b h_{0}^{2}}
$$

and

$$
M_{\mathrm{cr}}=T \eta h_{0}=\sigma_{s} A_{s} \eta h_{0} \Rightarrow \sigma_{s}=\frac{M_{\mathrm{cr}}}{A_{s} \eta h_{0}}
$$

where $M_{\mathrm{cr}}$ is the bending moment at the cracked cross-section, $\sigma_{c}$ and $\sigma_{s}$ are the maximum stress of concrete in compression and the stress in the tensile reinforcement, $C$ and $T$ are the corresponding stress resultants, respectively, $h_{0}$ is the effective height of the cross-section and $\eta$ is the lever arm of the force couple; $\omega \sigma_{c}$ denotes the average magnitude of the compression stress and $\xi h_{0}$ the height of the compression area, respectively.

Considering a cracked RC beam segment under a short-term bending moment (Fig. 3(b)), the average curvature $\bar{\varphi}$ can be defined as

$$
\bar{\varphi}=\frac{\bar{\varepsilon}_{c}+\bar{\varepsilon}_{s}}{h_{0}}=\frac{\psi_{c, x} \varepsilon_{c, x}+\psi_{s, x} \varepsilon_{s, x}}{h_{0}}
$$

in which $\bar{\varepsilon}_{c}$ and $\bar{\varepsilon}_{s}$ are the absolute average strains of compressed concrete and tensile reinforcement of the beam segment, respectively; $\varepsilon_{c, x}$ and $\varepsilon_{s, x}$ are the strain values of concrete in compression and reinforcement in tension at the cross-section $x$, respectively. $\psi_{c, x}$ and $\psi_{s, x}$ are the coefficients of the uneven strain distribution of concrete in compression and of reinforcement in tension of the cross-section $x$. As the strains between the cracked and intact cross-sections are different, an average strain has been used for the segment. 
By considering the constitutive laws of compressive concrete and tensile reinforcement, $\varepsilon_{c}=$ $\sigma_{c} / \beta E_{c}$ and $\varepsilon_{s}=\sigma_{s} / E_{s}$, and introducing Eqs. (16) and (17), the following expression is obtained (considering the cracked cross-section $x_{i}$ ):

$$
\begin{aligned}
\bar{\varphi} & =\frac{\bar{\varepsilon}_{c}+\bar{\varepsilon}_{s}}{h_{0}}=\frac{\psi_{c, x_{i}}\left(\sigma_{c, x_{i}} / \beta E_{c}\right)+\psi_{s, x_{i}}\left(\sigma_{s, x_{i}} / E_{s}\right)}{h_{0}}=\frac{\psi_{c, x_{i}}\left(M_{\mathrm{cr}, x_{i}} / \omega \xi \eta b h_{0}^{2} \beta E_{c}\right)+\psi_{s, x_{i}} / \eta\left(M_{\mathrm{cr}, x_{i}} / E_{s} A_{s} h_{0}\right)}{h_{0}} \\
& =\frac{M_{\mathrm{cr}, x_{i}} / \zeta E_{c} b h_{0}^{2}+\psi_{s, x_{i}} / \eta\left(M_{\mathrm{cr}, x_{i}} / E_{s} A_{s} h_{0}\right)}{h_{0}},
\end{aligned}
$$

where $\zeta=\omega \xi \eta \beta / \psi_{c, x_{i}}$ is the average strain coefficient of the compressed concrete, $\beta$ represents a variable reduction factor to take into account the nonlinearity of concrete.

Provided that the segment length $L$ approximates to the section dimensions ( $L \approx b$ or $h$ ), the curvature $\varphi(x)$ of each section $x$ along the segment can be assumed as a constant $\varphi_{0}{ }^{1}$ equal to the average curvature, namely, $\bar{\varphi}=\varphi_{0}$. Additionally, the section curvature has a relationship with the section moment and stiffness, $\varphi=(M / E I)=(M / B)$. Thus, the remnant stiffness at the cracked section $x_{i}$ in this segment, $B_{\mathrm{cr}, x_{i}}$, can be deduced from Eq. (18):

$$
B_{\mathrm{cr}, x_{i}}=\frac{E_{s} A_{s} h_{0}^{2}}{\left(\psi_{s, x_{i}} / \eta\right)+\left(\alpha_{E} \rho / \zeta\right)}=\frac{E_{s} A_{s} h_{0}^{2}}{\left(\psi_{s, x_{i}} / 0.87\right)+0.2+6 \alpha_{E} \rho},
$$

where $\alpha_{E}=\left(E_{s} / E_{c}\right)$ is Young's modulus ratio of steel and concrete; $\rho=\left(A_{s} / b h_{0}\right)$ is the reinforcement ratio of the beam section; $\alpha_{E} \rho / \zeta$ can be expressed into $0.2+6 \alpha_{E} \rho$ for rectangular sections and $\eta$ is approximately equal to 0.87 in most of the short-term loading cases [19]; Meanwhile, the coefficient of uneven strain distribution of reinforcement in tension, $\psi_{s, x}$, at the cross-section $x$ can be adopted from [19]

$$
\psi_{s, x}=1.1-0.65 \frac{f_{\mathrm{tk}}}{\sigma_{\mathrm{sk}, x} \rho_{\mathrm{te}}},
$$

where $\sigma_{\mathrm{sk}, x}=\left(M_{\mathrm{sk}, x} / A_{s} \eta h_{0}\right)=\left(M_{\mathrm{sk}, x} / 0.87 A_{s} h_{0}\right)$ denotes the equivalent stress of tensile reinforcement considering the standard ensemble of load effects, $f_{\mathrm{tk}}$ denotes the standard axial tensile strength of concrete, $\rho_{\mathrm{te}}=\left(A_{s} / A_{\mathrm{te}}\right)=\left(A_{s} / 0.5 \times b h\right)$ is the reinforcement ratio with respect to the area of concrete in tension, $\psi_{s, x}$ indicates the participation level of the concrete between two cracks in resisting the bending moment. And from Eq. (20), it can be seen that a higher bending moment results in a higher $\sigma_{\mathrm{sk}, x}$ and so induces an increase of $\psi_{s, x}$, which results in the reduction of $B_{\mathrm{cr}, x}$, as can be seen from Eq. (19).

\subsection{Generalized bending stiffness of a cracked $R C$ beam segment}

In practice, the stiffness estimation of a cracked structural segment, rather than a section, is more significant in evaluating the remnant load capacities of damaged RC structures. However, no such formula is given in the existing codes for the design of RC structures. Therefore, this study attempts to propose a practical formula for approximately estimating the remnant stiffness of a cracked beam segment based on the damage model illustrated in Fig. 4 (only one crack is assumed in the segment for simplicity). It should be mentioned that the damage model is only applicable to the damaged structures under short-term moments having cracked sections with open cracks.

If it is assumed as previously stated that the curvature is constant for the beam segment $\left(\varphi(x)=\varphi_{0}\right)$, then the stiffness, $B(x)=E I(x)$, follows the same variation as the moment $M(x)$ :

$$
B(x)=\frac{M(x)}{\varphi(x)}=\frac{M(x)}{\varphi_{0}}=\gamma M(x)
$$

with $\gamma=1 / \varphi_{0}$ the slope factor. 

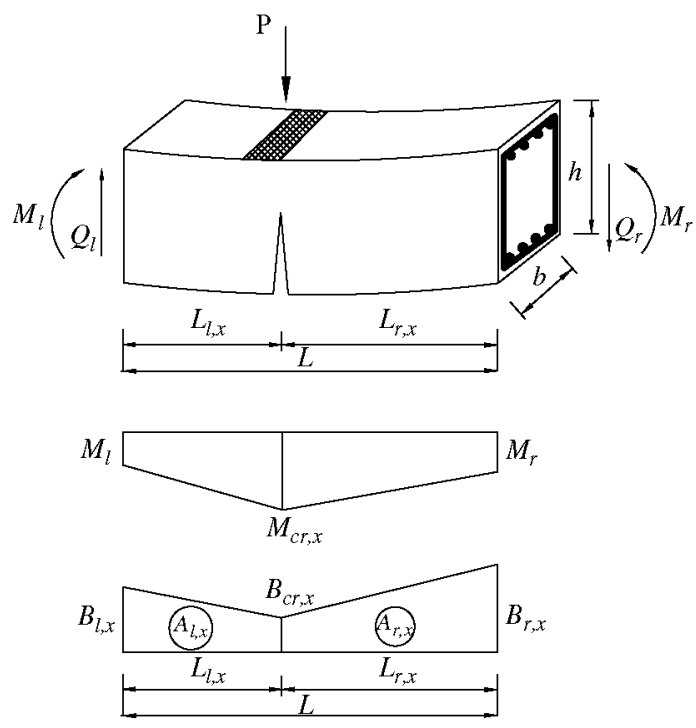

(Subsripts, $l$ and $r$, mean left side and right side, respectively)

Fig. 4. Damage model of an RC beam segment with one crack.

Then considering a linear variation for the moment, the remnant bending stiffness $B_{\text {re }}$ of the cracked beam segment can be formulated based on the proposed damage model (Fig. 4):

$$
\begin{aligned}
B_{\mathrm{re}}= & \frac{A_{l, x_{1}}+A_{r, x_{1}}+\cdots+A_{l, x_{i}}+A_{r, x_{i}}+\cdots+A_{l, x_{n}}+A_{r, x_{n}}}{L} \\
= & \frac{\left[\left(B_{\mathrm{cr}, x_{1}}+B_{l, x_{1}}\right) \times L_{l, x_{1}}+\left(B_{\mathrm{cr}, x_{1}}+B_{r, x_{1}}\right) \times L_{r, x_{1}}+\cdots+\left(B_{\mathrm{cr}, x_{n}}+B_{l, x_{n}}\right) \times L_{l, x_{n}}+\left(B_{\mathrm{cr}, x_{n}}+B_{r, x_{n}}\right) \times L_{r, x_{n}}\right]}{2 L} \\
& \left(L=L_{l, x_{1}}+L_{r, x_{1}}+\cdots+L_{l, x_{n}}+L_{r, x_{n}} ; n: \text { the number of the cracks in the segment }\right),
\end{aligned}
$$

where $B_{\mathrm{cr}, x_{i}}$ is the bending stiffness of the cracked section $x_{i}$, determined according to Eq. (19), and $B_{l / r, x_{i}}=$ $E_{c} I_{c}+E_{s} I_{s}$ is the stiffness of intact side cross-sections; $L_{l, x_{i}}$ and $L_{r, x_{i}}$ are the distances of the crack $x_{i}$ to the (left and right) edges of the segment $i$ and delimit the influence area of each crack, $A_{l, x_{i}}$ and $A_{r, x_{i}}$ are the areas under the stiffness versus distance relation at both sides of crack $x_{i}$. Once the reduced bending stiffness has been determined, the damage coefficient can be calculated with Eq. (15).

\section{Description of RC frame experiment}

Static and dynamic tests were performed on a laboratory-scale RC frame with one storey and one bay (Fig. 5). The geometric dimensions and reinforcement layout in the sections are illustrated in Fig. 5(b). Initially, the frame was tested dynamically with the purpose of determining the dynamic characteristics of the intact frame. Then a static concentrated load was applied at the mid span of the beam in a stepwise increasing way until the first visual crack appeared in the beam section adjacent to the loading point. And the static tests were performed with a simply supported boundary condition. Subsequently, a modal test was carried out on the cracked frame to obtain the dynamic characteristics of the damaged frame. By these means, modal parameters of the intact and damaged frame are available to validate the proposed model and method.

For the modal testing, the RC frame was supported on two rubber springs at the column bases in order to simulate a free-free boundary condition. Under this boundary condition, the cracks were opened, which can be advantageous for detecting the structural deterioration when the dynamic excitation is performed using 
(a)

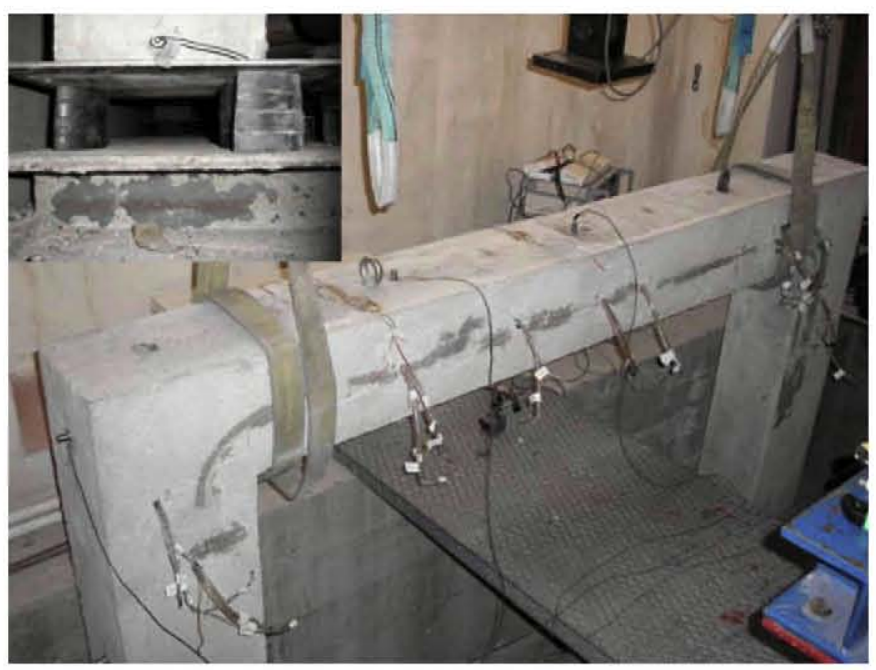

(b)

$\stackrel{0.275}{-}$

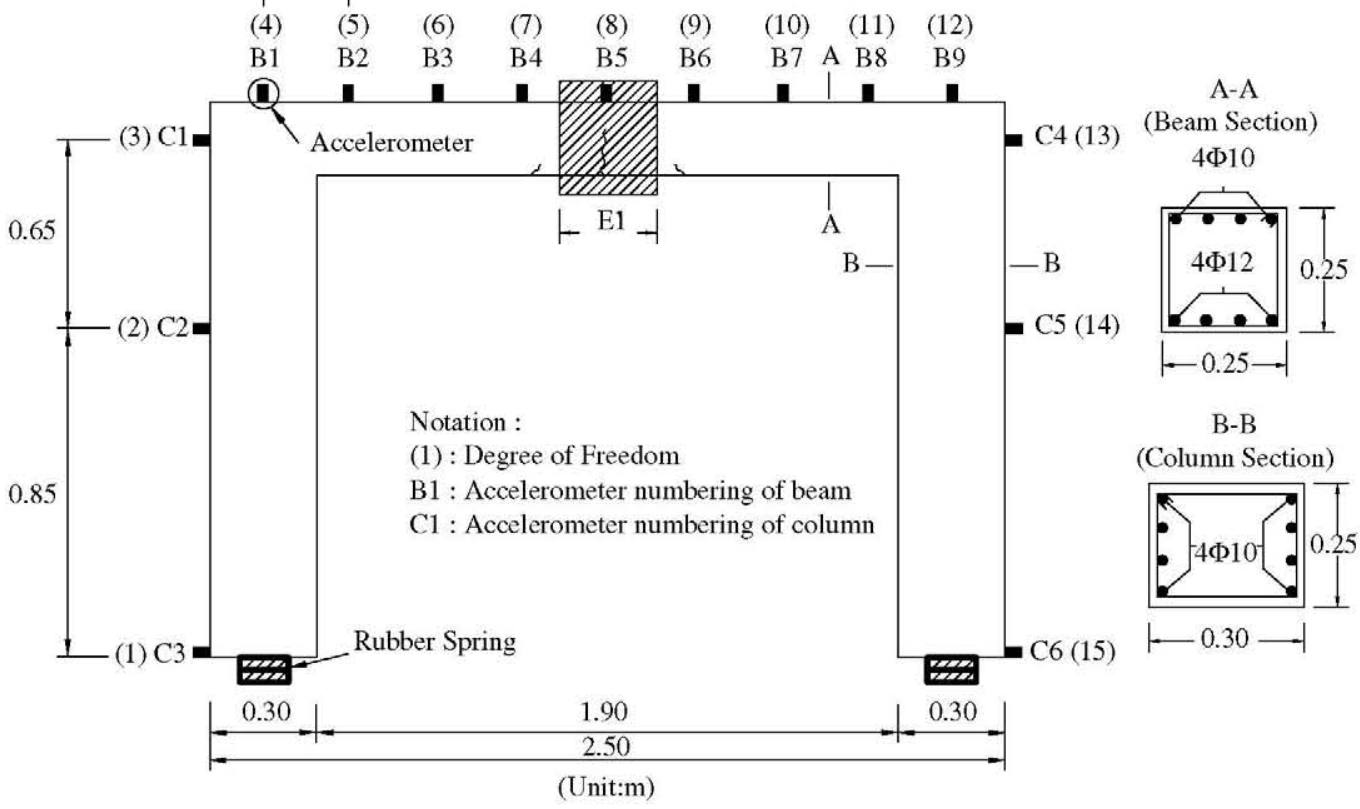

Fig. 5. Modal test of an RC frame: (a) In-situ modal test with spring boundary condition (top right corner). (b) Geometric dimensions and accelerometer arrangement.

low-energy devices. And the frame was excited by an impulsive load given by an impact hammer and the response was measured at different positions using piezoelectric accelerometers. The input and output signals were recorded and analyzed using a self-developed modal testing and analysis program IDAS and the modal frequencies and corresponding mass-normalized mode shapes were extracted from the measured data. Fig. 6(a) shows the first three experimental bending modes of the beam and it can be seen that the damage at the mid-span of beam causes a change in the mode shape. Table 1 presents a summary of the first nine modal frequencies of the intact and damaged frame. As expected, the frequencies decrease due to the damage. 


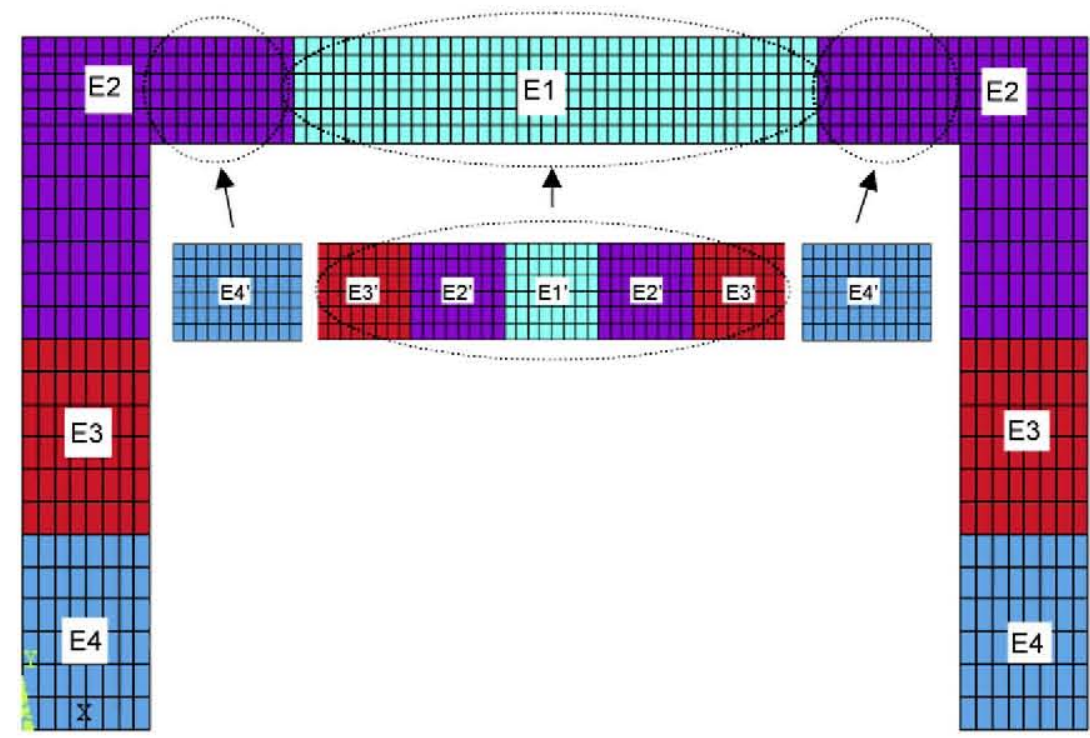

Fig. 7. Substructural division of an RC model.

FE model. A total of $n_{e}=756$ plane elements were generated, as shown in Fig. 7. And none of nodes in the FE model was constrained in order to simulate the free boundary condition close to the experimental boundary condition. Then a numeric modal analysis was carried out to obtain the first nine fundamental frequencies and the corresponding mode shapes.

For the updating problem, the analytical residual vector $\mathbf{r}$ comprises the first nine fundamental frequency residuals and 90 mode shape residuals of corresponding modes $(6 \times 15=90,6$ is the number of the adopted mode shapes and 15 is the number of the measured points, Fig. 5(b)), which means a total of $n_{r}=9+90=99$ residuals. But for the construction of sensitivity matrix, the first nine mode shapes are used resulting in $n_{r}^{\prime}=9+135=144$ numeric residuals.

\subsubsection{Updating procedures and performance of damage function}

The damage identification was performed in three updating steps. In the first step, the initially developed FE model was divided into four $\left(n_{p}=4\right)$ substructures, E1, E2, E3 and E4, as shown in Fig. 7. Then it was updated to the reference state using the measured modal frequencies and mode shapes of the undamaged frame. Subsequently, based on the reference model, the updating was repeated using the measured data of the damaged frame and the possibly damaged substructure (E1) was roughly identified. If a more detailed damage pattern was required, an additional updating step could be implemented. Only those substructures roughly detected in the second step would be updated again by dividing them into finer substructures. In our particular study, the damaged substructure $\mathbf{E} 1$ detected in the previous step was refined into three new substructures $\left(\mathbf{E} 1 \Rightarrow \mathbf{E} 1^{\prime}+\mathbf{E} 2^{\prime}+\mathbf{E} 3^{\prime}\right)$, and the remainder beam segments were also assigned a new substructure E4' in order to avoid misidentifying in the second step. Then Young's moduli of those new substructures were updated to accurately identify the damaged segment, E1'. In this sense, a rough estimation of possibly damaged substructure prior to an accurate identification avoids fractionizing the FE model into redundant substructures and thus improves the updating process and economizes the computational expense.

Concerning the performance of the bidimensional (step) damage function on the sensitivity matrix $\mathbf{J}$, using the reference updating process as an example, the original matrix $\mathbf{J}_{o}$ has a dimension of $n_{r} \times n_{e}=144 \times 756$ with a condition number of $3.263 \times 10^{18}$. But the dimension of the reduced matrix $\mathbf{J}_{r}$ is $n_{r} \times n_{p}=144 \times 4$ with a condition number 10.98 indicating a well-conditioned iterative problem. This proves that using damage function greatly improves the convergence performance of the optimization problem. 

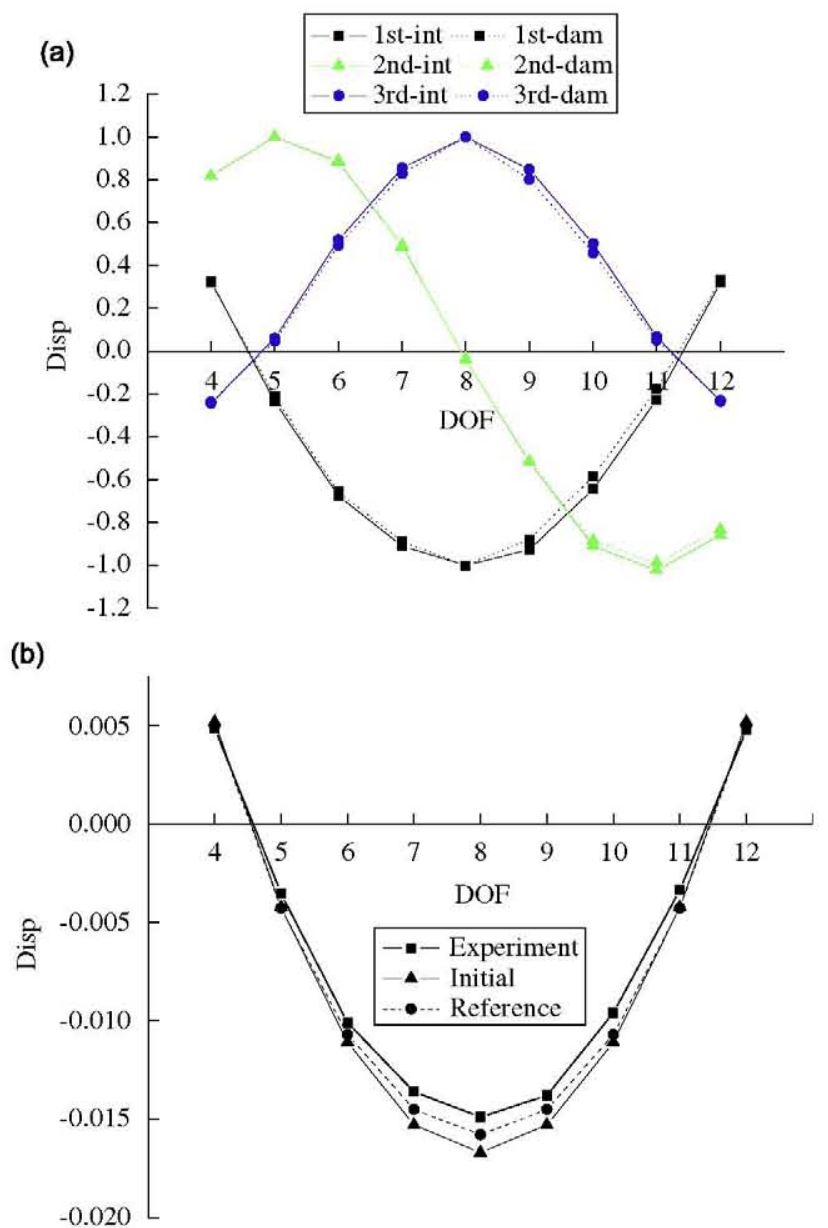

Fig. 6. Illustration of mode shapes of beam: (a) first three experimental mode shapes with unity normalization and (b) comparison of mass-normalized first mode after reference-state updating.

Table 1

Reduction factors of different substructures

\begin{tabular}{llclr}
\hline Substructure & $\mathbf{R}_{f, \text { ref }}$ & $\mathbf{R}_{f, \text { app }}$ & Substructure & $\mathbf{R}_{f, \text { dam }}$ \\
\hline E1 & -0.0734 & $\mathbf{0 . 1 2 4 1}$ & E1 & $\mathbf{0 . 3 8 0 4}$ \\
E2 & 0.1215 & 0.0401 & E2 & 0.1106 \\
E3 & -0.0667 & -0.0189 & E3 & 0.0650 \\
E4 & -0.1672 & -0.0064 & E4 & -0.0117 \\
\hline
\end{tabular}

Note: $\mathbf{R}_{f, \text { ref }}, \mathbf{R}_{f, \text { app }}$ and $\mathbf{R}_{f \text {,dam }}$ denote the stiffness reduction factors of the reference state, the approximate estimation step and the damage state, respectively.

\section{Damage identification of an $\mathrm{RC}$ frame}

\subsection{Damage identification by FE updating}

\subsubsection{FE modeling and residual construction}

A 2D plane element having four nodes with two translation degrees of freedom at each node was used to model the RC frame. The measured material properties and geometrical dimensions were used to develop the 
Table 2

Updated modal frequencies of reference and damage states

\begin{tabular}{|c|c|c|c|c|c|c|c|c|c|c|}
\hline \multirow{2}{*}{$\begin{array}{l}\text { Updating } \\
\text { state } \\
\text { Mode }\end{array}$} & \multicolumn{5}{|c|}{ Undamaged } & \multicolumn{5}{|c|}{ Damaged } \\
\hline & $\begin{array}{l}\operatorname{Exp} \\
(\mathrm{Hz})\end{array}$ & $\begin{array}{l}\text { FEA }_{\text {Initial }} \\
(\mathrm{Hz})\end{array}$ & $\begin{array}{l}\text { Error } \\
(\%)\end{array}$ & $\begin{array}{l}F_{E A_{\text {ref }}} \\
(\mathrm{Hz})\end{array}$ & $\begin{array}{l}\text { Error } \\
(\%)\end{array}$ & $\begin{array}{l}\operatorname{Exp} \\
(\mathrm{Hz})\end{array}$ & $\begin{array}{l}F E A_{\text {ref }} \\
(\mathrm{Hz})\end{array}$ & $\begin{array}{l}\text { Error } \\
(\%)\end{array}$ & $\begin{array}{l}\text { FEA }_{\text {dam }} \\
(\mathrm{Hz})\end{array}$ & $\begin{array}{l}\text { Error } \\
(\%)\end{array}$ \\
\hline 1 & 30.16 & 30.58 & 1.40 & 30.23 & 0.22 & 29.1 & 30.23 & 3.87 & 29.07 & -0.11 \\
\hline 2 & 69.34 & 71.36 & 2.91 & 68.52 & -1.18 & 68.05 & 68.52 & 0.69 & 66.91 & -1.67 \\
\hline 3 & 178.11 & 183.24 & 2.88 & 180.78 & 1.50 & 170.24 & 180.78 & 6.19 & 174.94 & 2.76 \\
\hline 4 & 339.06 & 336.87 & -0.65 & 336.18 & -0.85 & 335.53 & 336.18 & 0.19 & 330.60 & -1.47 \\
\hline 5 & 348.74 & 349.29 & 0.16 & 347.88 & -0.25 & 344.65 & 347.88 & 0.94 & 342.47 & -0.63 \\
\hline 6 & 509.78 & 528.19 & 3.61 & 525.15 & 3.01 & 499.53 & 525.15 & 5.13 & 511.78 & 2.45 \\
\hline 7 & 709.86 & 685.99 & -3.36 & 695.50 & -2.02 & 679.41 & 695.50 & 2.37 & 669.96 & -1.39 \\
\hline 8 & 790.07 & 788.76 & -0.17 & 781.73 & -1.06 & 768.7 & 781.73 & 1.70 & 760.44 & -1.07 \\
\hline 9 & 948.13 & 946.59 & -0.16 & 948.08 & -0.01 & 933.98 & 948.08 & 1.51 & 937.96 & 0.43 \\
\hline
\end{tabular}

(a)
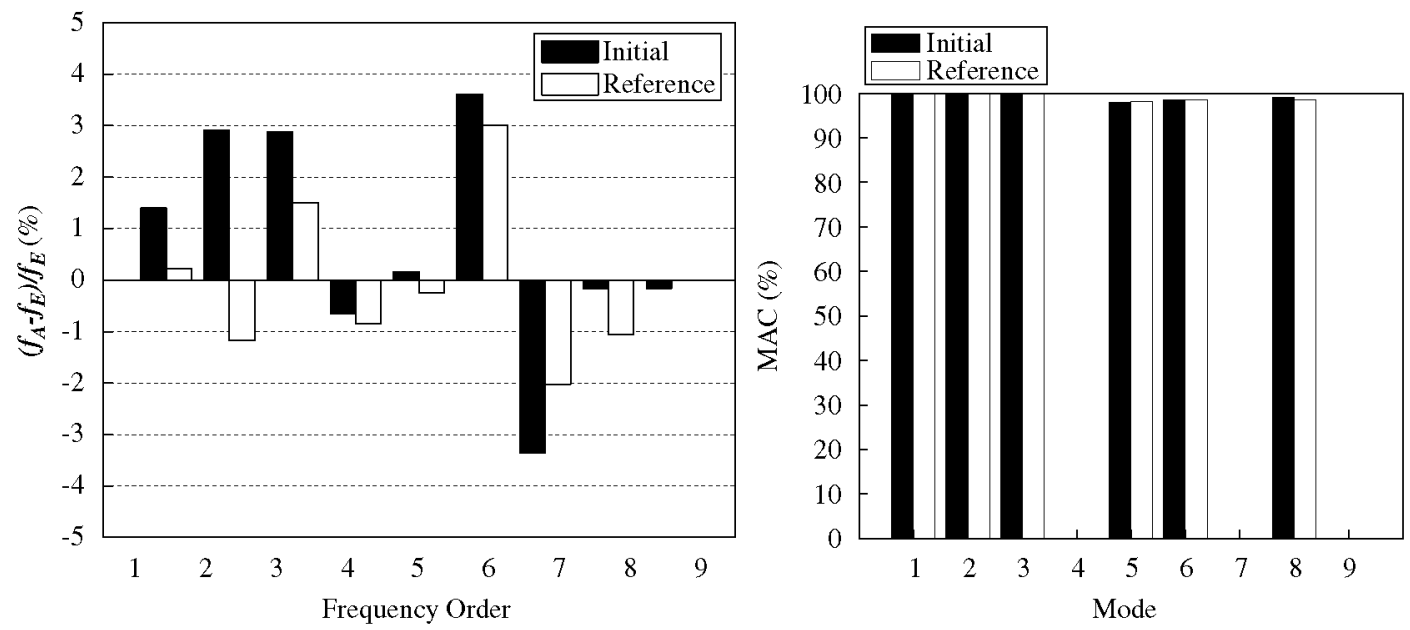

(b)
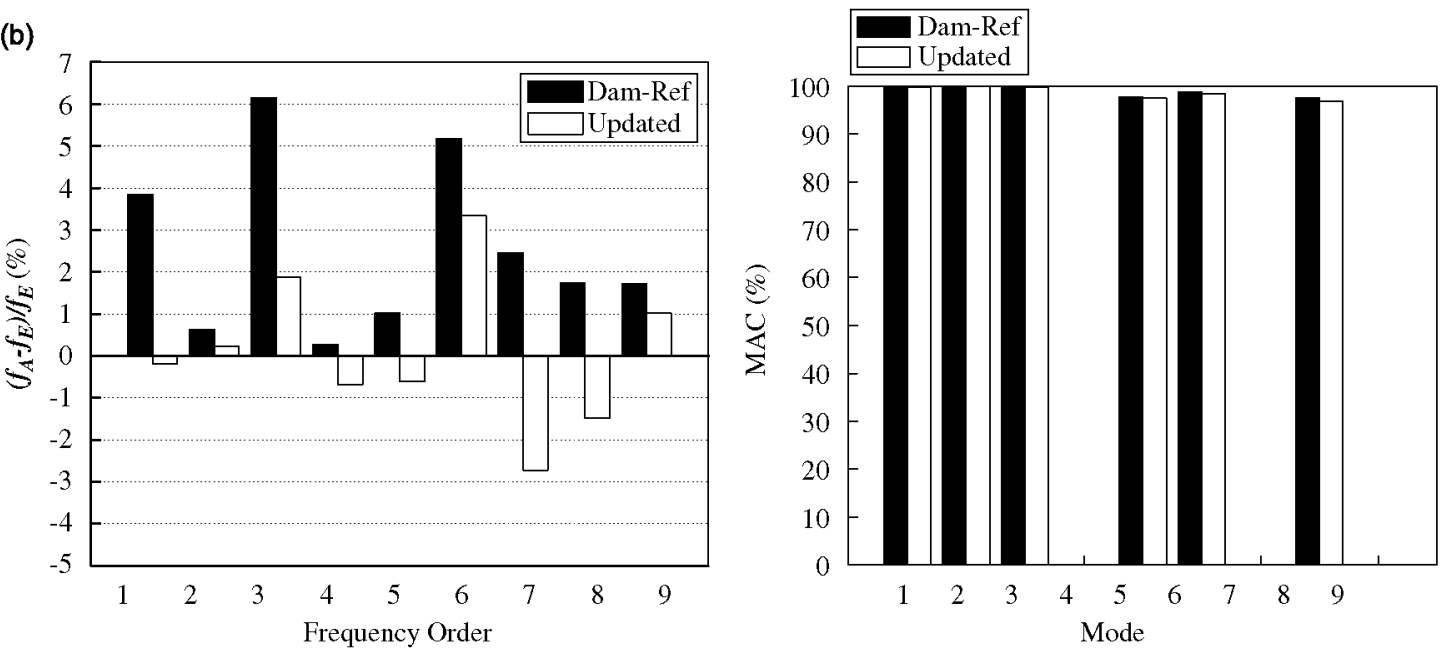

Fig. 8. Illustration of updated modal parameters: (a) comparison of initial and reference updating states and (b) comparison of reference and damage updating states. 


\subsubsection{Reference state updating}

Young's moduli of the four substructures, E1, E2, E3 and E4, were updated separately to minimize the discrepancies between the experimental and numerical modal data. The initial FE model was updated to the reference state with the updating results shown in Tables 1 and 2, Figs. 6 and 8. Table 2 lists the relative differences in modal frequencies with respect to the experimental results both for the initial and updated FE models. It is evident that a clear improvement appears for most frequencies after updating. As can be observed in Fig. 8, only minor differences appear in the MAC-values between the initial and the updated models, since very small changes occur for the experimental mode shapes (Fig. 6(a)). Meanwhile, Table 1 shows that all Young's moduli increase (negative correction factors) except that of the substructure at joint region (E2), which decreases (positive correction factors) about $12 \%$ with respect to the initial value. This is reasonable due to the fact that when casting, the concrete in the joint regions was not so compact as that of the concrete specimens. On the contrary, the existence of reinforcement contributes to the increase of the stiffness of the other substructures (for substructure $\mathbf{E} 2$, the contribution of reinforcement could not compensate the stiffness reduction caused by casting).

\subsubsection{Damage state updating}

The identification of the applied damage was performed in two steps. Firstly, the reference FE model was updated again using the measured modal data of the damaged frame. The same optimization procedure with the same residuals as in the reference updating was applied and Table 1 clearly indicates that $\mathbf{E} 1$ was seriously damaged when compared with the other substructures. However, upon considering only four substructures for the whole frame the damage identification could be very rough since the possible damage is supposed to spread over a limited zone. Hence, in order to accurately locate the damage, E1 was refined into three new substructures, E1', E2' and E3' (Fig. 7, E4' was included as a supplement due to the consideration of updating the whole beam), and the updating was repeated based on the reference FE model but only Young's moduli of the four new substructures were adjusted to accurately locate and quantify the damage occurred in $\mathbf{E} 1^{\prime}$ and $\mathbf{E} 2^{\prime}$ (Table 1), which agree with the experimental observations. Furthermore, the substructure $\mathbf{E} 1^{\prime}$ has a stiffness reduction of $38 \%$ with respect to the reference value and $\mathbf{E} 2{ }^{\prime}$ of $11 \%$ might also be lightly damaged, which is also in agreement with the experimental results.

To evaluate the consistency between the coarse mesh of damage elements and the refined mesh, an equivalent stiffness reduction factor or damage coefficient $d_{\mathrm{E} 1 \text {,eq }}$ was calculated for $\mathbf{E} 1=\mathbf{E} 1^{\prime}+\mathbf{E} 2^{\prime}+\mathbf{E} 3^{\prime}$ as follows:

$$
d_{\mathrm{E} 1, \mathrm{eq}}=d_{\mathbf{E} 1^{\prime}} \frac{L_{\mathbf{E} 1^{\prime}}}{L_{\mathbf{E} 1}}+d_{\mathbf{E} 2^{\prime}} \frac{L_{\mathbf{E} 2^{\prime}}}{L_{\mathbf{E} 1}}+d_{\mathbf{E} 3^{\prime}} \frac{L_{\mathbf{E} 3^{\prime}}}{L_{\mathrm{E} 1}}=0.3804 \times 0.2+0.1106 \times 0.4+0.0650 \times 0.4=0.1463 .
$$

If compared with the value of the damage coefficient for the substructure E1 (Table 2), which is 0.1241, the following relative difference can be obtained:

$$
\operatorname{diff}=\left|\frac{d_{\mathrm{E} 1}-d_{\mathrm{E} 1, \mathrm{eq}}}{d_{\mathrm{E} 1}}\right|=\left|\frac{0.1241-0.1463}{0.1241}\right| 100 \%=18 \% .
$$

\subsection{Damage identification using damage model}

The damage coefficient can also be evaluated using the damage model proposed in Section 3. In this case study, the cracked segment $\mathbf{E} 1$ ' having a length of $L=24 \mathrm{~cm}(b=h=25 \mathrm{~cm})$ is analyzed. Then the remnant bending stiffness $B_{\text {re }}$ can be calculated using Eqs. (19) and (22):

$$
\begin{aligned}
B_{\mathrm{cr}, x_{\mathrm{E} 1^{\prime}}}=\frac{E_{s} A_{s} h_{0}^{2}}{\left(\psi_{s, x_{\mathrm{E} 1^{\prime}}} / 0.87\right)+0.2+6 \alpha_{E} \rho}=\frac{2.1 \times 10^{11} \times 452 \times 10^{-6} \times(0.9 \times 0.25)^{2}}{(0.72 / 0.87)+0.2+6 \times 7.36 \times 0.008}=3.48 \times 10^{6}\left(\mathrm{~N} \mathrm{~m}^{-1}\right), \\
B_{l, x_{\mathrm{E} 1^{\prime}}}=B_{r, x_{\mathrm{E} 1^{\prime}}}=\left(1-R_{f, \mathbf{E} 2^{\prime}}\right) B_{\text {intact }}=(1-0.1106) \times 10.68 \times 10^{6}=9.50 \times 10^{6}\left(\mathrm{~N} \mathrm{~m}^{-1}\right) .
\end{aligned}
$$


Thus,

$$
\begin{aligned}
B_{\mathrm{re}} & =\frac{A_{l, x_{\mathrm{E} 1^{\prime}}}+A_{r, x_{\mathrm{E} 1^{\prime}}}}{L}=\frac{1}{L}\left[\frac{\left(B_{c r, x_{\mathrm{E} 1^{\prime}}}+B_{l, x_{\mathrm{E} 1^{\prime}}}\right) L / 2}{2}+\frac{\left(B_{\mathrm{Cr}, x_{\mathrm{E} 1^{\prime}}}+B_{r, x_{\mathrm{E} 1^{\prime}}}\right) L / 2}{2}\right] \\
& =\frac{1}{4}[2(3.48+9.50)] 10^{6}=6.49 \times 10^{6}\left(\mathrm{~N} \mathrm{~m}^{-1}\right) .
\end{aligned}
$$

\subsection{Comparison of the stiffness reductions predicted by the FE updating method and the damage model}

It should be mentioned herein that the remnant stiffness calculated using the proposed damage model is based on a static mechanism, but the updated stiffness by FE updating is actually based on a dynamic mechanism, which means a direct stiffness comparison is not appropriate. Hence, a nondimensional reduction factor $R_{f}$ is proposed for the comparison. Then the stiffness reduction calculated using the damage model is

$$
R_{f, \text { static }}=1-\frac{B_{\mathrm{re}}}{B_{l, x}}=1-\frac{6.49 \times 10^{6}}{10.68 \times 10^{6}} \times 100 \%=39.2 \% .
$$

And the reduction of dynamic stiffness of $\mathbf{E} 1, R_{f}$,dynamic, is $38 \%$ given in Table 1 . It is found that $R_{f \text {, dynamic }}$ is very close to $R_{f, \text { static }}$ with a difference of only $1.2 \%$, which proves that the damage model is successfully verified and can be used in similar calculations.

\section{Conclusions}

Damage identification of a tested RC frame by a sensitivity-based FE model updating method and an RC damage model is presented in this paper. The updating method aims to adjust the FE model of the RC frame as closely as possible to the experimental model by tuning Young's modulus of concrete. The frame model is divided into several substructures and Young's moduli of the different substructures are updated by minimizing the discrepancies of the experimental and numeric modal frequencies and mass-normalized mode shapes. A bidimensional damage function developed for 2D FE models is proposed to reduce the number of unknown updating variables. And together with the trust region algorithm, the application of the damage function results in a well-conditioned optimization problem and considerably improves the convergence performance. It is found that the proposed updating method can identify the damage located at the mid span of the beam.

At the same time, an $\mathrm{RC}$ damage model in a static mechanism is also proposed to estimate the remnant bending stiffness of a cracked beam segment under a short-term bending moment. The feasibility of the damage model is verified against the updating results and it is shown that the proposed damage model is applicable to an approximate estimation of the remnant bending stiffness of a cracked RC beam segment.

\section{References}

J.M.W. Brownjohn, Structural health monitoring of civil infrastructure, Philosophical Transactions of the Royal Society A 365 (1851) (2007) 589-622.

J.E. Doherty, Nondestructive evaluation, in: A.S. Kobayashi (Ed.), Handbook on Experimental Mechanics, Society for Experimental Mechanics, Inc., 1987 (Chapter 12).

S.W. Doebling, C.R. Farrar, M.B. Prime, A summary review of vibration-based damage identification methods, The Shock and Vibration Digest 30 (2) (1998) 91-105.

W.X. Ren, G.D. Roeck, Structural damage identification using modal data, I: simulation verification, Journal of Structural Engineering-ASCE 128 (1) (2002) 87-95.

W.X. Ren, G.D. Roeck, Structural damage identification using modal data, II: test verification, Journal of Structural EngineeringASCE 128 (1) (2002) 96-104.

Y.J. Yan, L. Cheng, Z.Y. Wu, L.H. Yam, Development in vibration-based structural damage detection technique, Mechanical Systems and Signal Processing 21 (5) (2007) 2198-2211. 
R. Perera, R. Torres, Structural damage detection via modal data with genetic algorithms, Journal of Structural Engineering-ASCE 132 (9) (2006) 1491-1501.

N.D. Biswa, Finite element model updating, eigenstructure assignment and eigenvalue embedding techniques for vibrating systems, Mechanical Systems and Signal Processing 16 (1) (2001) 83-96 ((Invited Paper), Special Issue on Vibration Control).

B. Jaishi, W.X. Ren, Damage detection by finite element model updating using modal flexibility residual, Journal of Sound and Vibration 290 (2006) 369-387.

M.I. Friswell, Damage identification using inverse methods, Philosophical Transactions of the Royal Society A 365 (1851) (2007) 393-410.

M.M. Gola, A. Somae, D. Botto, On theoretical limits of dynamic model updating using a sensitivity-based approach, Journal of Sound and Vibration 244 (4) (2001) 583-595.

J. Nocedal, S.J. Wright, Numerical Optimization, Springer, New York, 1999.

M.I. Friswell, J.E. Mottershead, Finite Element Model Updating in Structural Dynamics, Kluwer Academic Publishers, Dordrecht, The Netherlands, 1995.

M. Link, Updating of analytical models - review of numerical procedures and application aspects, Proceedings of the Structural Dynamics Forum SD2000, Los Alamos, USA, 1999.

B. Jaishi, W.X. Ren, Structural finite element model updating using ambient vibration test results, Journal of Structural EngineeringASCE 131 (4) (2005) 617-628.

A. Teughels, J. Maeck, G. De Roeck, Damage assessment by FE model updating using damage functions, Computers and Structures 80 (25) (2002) 1869-1879.

Matlab $^{\mathbb{\circledR}}$ rev. 6.5 (release 13), MathWorks Inc., Natick, MA, USA, 2002.

R. Fox, M. Kapoor, Rate of change of eigenvalues and eigenvectors, AIAA Journal 6 (12) (1968) 2426-2429.

Chinese Standard GB50010, Code for Design of Concrete Structures, Department of the Ministry of Construction, Beijing, PR China, 2002.

IDAS, A Data Acquisition and Analysis Program for Modal Test, Department of Structural Mechanics and Industrial Constructions, ETSII, Universidad Politécnica de Madrid, Madrid, Spain, 2000. 